\title{
Comparison of Some Methods for Solving the Internal Wave Propagation Problem in a Weakly Stratified Fluid
}

\author{
M. N. Moskalkov ${ }^{a}$ and D. Utebaev ${ }^{b}$ \\ ${ }^{a}$ National Aviation University, pr. Kosmonavta Komarova 1, Kiev, 03680 Ukraine \\ ${ }^{b}$ Berdakh Kara-Kalpak State University, ul. Akad. Abdirov 1, Nukus, 742000 Uzbekistan \\ Received June 19, 2009
}

\begin{abstract}
Three difference schemes for solutions of problems of internal wave fluid dynamics are put forward and examined using the finite difference and finite element methods. The schemes proposed have been tested numerically and subjected to comparative analysis.
\end{abstract}

Keywords: internal waves, finite difference method, weakly stratified fluid.

DOI: $10.1134 /$ S2070048211020086

\section{INTRODUCTION}

The problem mentioned in the title arises naturally in atmospheric physics, ocean science, theory of rotating fluids, etc. As pointed out in [1], only a numerical experiment is capable of providing sufficient information as to the details of the motion of internal waves. However, even numerical calculations have their own limitations determined by stability, accuracy and efficiency. Consequently, it is a matter of practical importance to work out a method that is optimal from this standpoint. Here, we aim to make such an attempt.

\section{STATEMENT OF THE PROBLEM}

Consider the problem

$$
\begin{aligned}
\frac{\partial^{2}}{\partial t^{2}}(L u)+\omega_{0}^{2} L_{1} u & =-f(x, t), \quad(x, t) \in Q_{T}=\left\{x \in \Omega \subset R^{2}, t \in(0, T]\right\}, \\
u & =0, \quad x \in \Gamma=\partial \Omega, \quad t \in(0, T], \\
u(x, 0) & =u_{0}(x), \quad \frac{\partial u}{\partial t}(x, 0)=u_{1}(x), \quad x \in \bar{\Omega} .
\end{aligned}
$$

Here, $L u=\frac{\partial^{2} u}{\partial x_{1}^{2}}+\frac{\partial^{2} u}{\partial x_{2}^{2}}, L_{1} u=\frac{\partial^{2} u}{\partial x_{1}^{2}}$, and $\Omega=\left\{0 \leq x_{p} \leq l_{p}, p=1,2\right\}$. A (weak) generalized solution of the problem is, by definition, a function $u(x, t) \in H=\stackrel{\circ}{W}_{2}^{1}(\Omega)$, which has the partial derivative $\frac{\partial^{2} u}{\partial t^{2}} \in W_{2}^{1}(\Omega)$ for each $t \in[0, T]$ and which satisfies relations [1] almost everywhere:

$$
a\left(\frac{d^{2} u}{d t^{2}}(t), \vartheta\right)+\omega_{0}^{2} a_{1}(u(t), \vartheta)=(f(t), \vartheta), \quad \forall \vartheta \in H, \quad u(0)=u_{0}, \quad \frac{d u}{d t}(0)=u_{1} .
$$

Here,

$$
a(\vartheta, \vartheta)=\sum_{m=1}^{2} a_{m}(\vartheta, \vartheta)=\|\vartheta\|_{1}^{2}>0
$$




$$
a_{m}(\vartheta, \vartheta)=\iint_{\Omega}\left(\frac{\partial \vartheta}{\partial x_{m}}\right)^{2} d x=\left\|\frac{\partial \vartheta}{\partial x_{m}}\right\|^{2} \geq 0, \quad m=1,2, \quad \forall \vartheta \in H,
$$

and $u(t)$ is a function of abstract argument $t \in[0, T]$ with values in $H$. See [1] for the existence of a solution to this problem.

\section{APPROXIMATION IN SPACE}

We construct a subspace $H_{h} \subset H$ to approximate $H$. Suppose that the operators $D, A: H_{h} \longrightarrow H_{h}$ approximate the operators $-L$ and $-\omega_{0}^{2} L_{1}$, respectively. We replace problem (1)-(3) by the Cauchy problem for a function of abstract argument $u_{h}(t) \in H_{h}$,

$$
D \frac{d^{2} u_{h}(t)}{d t^{2}}+A u_{h}(t)=f_{h}(t), \quad u_{h}(0)=u_{0, h}, \quad \frac{d u_{h}}{d t}(0)=u_{1, h} .
$$

Here, $u_{m, h}=P_{h} u_{m}, m=0,1$ are interpolants to the initial conditions, and $P_{h}$ is the projection operator $P_{h}: H \longrightarrow H_{h}$ and $f_{h}(t)=P_{h} f(t)$.

Two ways shall be considered for selecting subspace $H_{h}$.

The first way corresponds to the difference approximation of Eq. (1) in space variables. Consider the grid $\bar{\omega}_{h}=\bar{\omega}_{h_{1}} \times \bar{\omega}_{h_{2}}$, where $\bar{\omega}_{h_{m}}=\left\{x_{m}=i_{m} h_{m}, i_{m}=\overline{0, N_{m}}, h_{m}=l_{m} / N_{m}\right\}, m=1,2$. In this case, the subspace

is as follows: $H_{h}=\stackrel{\circ}{W}_{2}^{1}\left(\omega_{h}\right)$ (see [2]). Approximating the operators $-L$ and $-\omega_{0}^{2} L_{1}$ on the grid $\omega_{h}$ by central difference derivatives [2], it follows that

$$
D_{V}=-v_{x_{1} \bar{x}_{1}}-v_{x_{2} \bar{x}_{2}}, \quad A V=-\omega_{0}^{2} v_{x_{1} \bar{x}_{1}}
$$

Here $v_{x_{1} \bar{x}_{1}}=\left(v_{i_{1}+1, i_{2}}-2 v_{i_{1} i_{2}}+v_{i_{1}-1, i_{2}}\right) / h_{1}^{2}, v_{x_{2} \bar{x}_{2}}=\left(v_{i_{1}, i_{2}+1}-2 v_{i_{1} i_{2}}+v_{i_{1}, i_{2}-1}\right) / h_{2}^{2}, v_{i_{1} i_{2}}=v\left(i_{1} h_{1}, i_{2} h_{2}, t\right)$.

The second way corresponds to the approximation of Eq. (1) in space variables by the finite element method. We subdivide the domain $\Omega$ into $N_{1} \times N_{2}$ rectangles: $\Omega_{i j}=\left\{(i-1) h_{1} \leq x_{1} \leq i h_{1},(j-1) h_{2} \leq x_{2} \leq j h_{2}\right\}$, $i=\overline{1, N_{1}}, j=\overline{1, N_{2}}$, and $h_{m}=\frac{l_{m}}{N_{m}}$.

Consider the following system of basis functions

$$
\varphi_{i j}\left(x_{1}, x_{2}\right)=\varphi_{i}\left(x_{1}\right) \varphi_{j}\left(x_{2}\right), \quad i=\overline{1, N_{1}-1}, \quad j=\overline{1, N_{2}-1},
$$

where $\varphi_{i}(x)=B_{3}\left(\frac{x-x_{i}}{h}\right), i=\overline{2, N-2}, B_{3}(\xi)$ is the cubic $B$-spline [3]:

$$
\begin{gathered}
B_{3}(\xi)= \begin{cases}(2-|\xi|)^{3}, & 0 \leq|\xi| \leq 2, \\
1+3(1-|\xi|)+3(1-|\xi|)^{2}-3(1-|\xi|)^{3}, & |\xi| \leq 1, \\
0, & |\xi|>2,\end{cases} \\
\varphi_{1}(x)=B_{3}^{-}\left(\frac{x-x_{1}}{h}\right), \quad B_{3}^{-}(\xi)= \begin{cases}2\left(2-2 \xi-\xi^{2}\right)(1+\xi), & -1 \leq \xi \leq 0, \\
1+3(1-\xi)+3(1-\xi)^{2}-3(1-\xi)^{3}, & 0 \leq \xi \leq 1, \\
(2-\xi)^{3}, & 1 \leq \xi \leq 2, \\
0 & \xi>2,\end{cases}
\end{gathered}
$$




$$
\varphi_{N-1}(x)=B_{3}^{+}\left(\frac{x-x_{N-1}}{h}\right), \quad B_{3}^{+}(\xi)= \begin{cases}2\left(2+\xi-\xi^{2}\right)(1-\xi), & 0 \leq \xi \leq 1, \\ 1+3(1+\xi)+3(1+\xi)^{2}-3(1+\xi)^{3}, & -1 \leq \xi \leq 0, \\ (2+\xi)^{3}, & -2 \leq \xi \leq-1, \\ 0 & \xi<0 .\end{cases}
$$

The reason for these special functions for $i=1$ and $i=N-1$ is that the homogeneous boundary conditions of the first kind must be satisfied. In constructing these functions, it has also been taken into account that all $\varphi_{i}(x) \in C^{2}[0, l]$.

Now, the approximate solutions can be written in the form of a bicubic spline

$$
u_{h}\left(x_{1}, x_{2}, t\right)=\sum_{k=1}^{N} a_{k}(t) \varphi_{k}\left(x_{1}, x_{2}\right),
$$

where $\varphi_{k}\left(x_{1}, x_{2}\right)=\varphi_{i}\left(x_{1}\right) \varphi_{j}\left(x_{2}\right), i=\overline{1, N_{1}-1}, j=\overline{1, N_{2}-1}$, and $k=i+(j-1)\left(N_{1}-1\right), N=\left(N_{1}-1\right)\left(N_{2}-1\right)$. The space $H_{h}$ consists of elements of the form (7).

The stiffness matrices, which correspond to the operators $D$ and $A$, are calculated as follows:

$$
\mathbf{D}=\left\{a\left(\varphi_{k}\left(x_{1}, x_{2}\right), \varphi_{m}\left(x_{1}, x_{m}\right)\right)\right\}_{k, m=1}^{N}, \quad \mathbf{A}=\left\{\omega_{0}^{2} a_{1}\left(\varphi_{k}\left(x_{1}, x_{2}\right), \varphi_{m}\left(x_{1}, x_{2}\right)\right)\right\}_{k, m=1}^{N},
$$

where $k=i+(j-1)\left(N_{1}-1\right), m=p+(q-1)\left(N_{1}-1\right) ; i, p=\overline{1, N_{1}-1}, j, p=\overline{1, N_{2}-1}$.

For both approaches,

$$
D=D^{*}>0, \quad A=A^{*}>0 \text { and } \omega_{0}^{2} D \geq A
$$

\section{APPROXIMATION IN TIME}

We again consider two approaches.

Under the first way, a three-layer difference scheme with central differences is used for discretization of problem (5):

$$
\begin{gathered}
D \frac{y^{n+1}-2 y^{n}+y^{n-1}}{\tau^{2}}+A y^{n}=f_{h}\left(t_{n}\right), \quad y^{n} \in H_{h}, \quad t_{n} \in \omega_{\tau}, \\
y^{0}=u_{0, h}, \quad D \frac{y^{1}-y^{0}}{\tau}=u_{1, h}+\frac{\tau}{2}\left(f_{h}^{0}-A y^{0}\right) .
\end{gathered}
$$

Here, $y^{n}=y\left(t_{n}\right), \omega_{\tau}=\left\{t_{n}=n \tau, n=1,2, \ldots, \tau>0\right\}$.

The second approach was put forward in [4, 5], where, in order to solve problem (5), the finite element method with respect to variable $t$ and involving cubical Hermitean elements (see formula (15)) was used to build a two-layer two-parameter vector difference scheme, which binds $\dot{y}^{n+1}, \dot{y}^{n}, y^{n+1}$, and $y^{n}$, which in turn approximate the derivatives $\frac{d u_{h}}{d t}\left(t_{n}+\tau\right), \frac{d u_{h}}{d t}\left(t_{n}\right), u_{h}\left(t_{n}+\tau\right)$, and $u_{h}\left(t_{n}\right)$, respectively:

$$
\begin{gathered}
\left(D-\frac{1}{12} \tau^{2} A\right) \frac{\dot{y}^{n+1}-\dot{y}^{n}}{\tau}+A \frac{y^{n+1}+y^{n}}{2}=\Phi_{1}, \\
\left(D-\alpha \tau^{2} A\right) \frac{y^{n+1}-y^{n}}{\tau}-\left(D-\beta \tau^{2} A\right) A \frac{\dot{y}^{n+1}+\dot{y}^{n}}{2}=\Phi_{2}, \\
y(0)=u_{h, 0}, \quad \dot{y}(0)=u_{h, 1} .
\end{gathered}
$$


Here,

$$
\begin{gathered}
\Phi_{p}=\frac{1}{\tau} \int_{t_{n}}^{t_{n+1}} f(t) \vartheta_{p}\left(\frac{t-t_{n}}{\tau}\right) d t=\int_{0}^{1} f\left(t_{n}+\tau \xi\right) \vartheta_{p}(\xi) d \xi, \quad p=1,2, \\
\vartheta_{1}(\xi)=1, \quad \vartheta_{2}(\xi)=s_{1} \tau(\xi-0.5)+s_{2} \tau\left(\xi^{3}-1.5 \xi^{2}+0.5 \xi\right),
\end{gathered}
$$

and parameters $s_{1}, s_{2}$ are determined from the relations: $s_{1}=180 \beta-40 \alpha$ and $s_{2}=1680 \beta-280 \alpha$.

From the calculated values of $\dot{y}^{n+1}, \dot{y}^{n}, y^{n+1}$, and $y^{n}$ it is possible to restore the approximation to $u_{h}(t)$ for any $t \in\left[t_{n}, t_{n+1}\right], n=0,1, \ldots$ by the formula

$$
y(t)=y^{n} \varphi_{00}^{n}(t)+\dot{y}^{n} \varphi_{10}^{n}(t)+y^{n+1} \varphi_{01}^{n}(t)+\dot{y}^{n+1} \varphi_{11}^{n}(t) .
$$

Here, $\varphi_{00}^{n}(t)=2 \xi^{3}-3 \xi^{2}+1, \varphi_{01}^{n}(t)=3 \xi^{2}-2 \xi^{3}, \varphi_{10}^{n}(t)=\tau\left(\xi^{3}-2 \xi^{2}+\xi\right), \varphi_{11}^{n}(t)=\tau\left(\xi^{3}-\xi^{2}\right), \xi=\frac{t-t_{n}}{\tau}$.

Combining the approximation in space and time, we consider three schemes to solve problem (1)-(3):

Scheme $\mathbf{1}^{\mathbf{0}}$ : Difference approximation of the second order of accuracy, as taken in space (6) and in time (10)-(11);

Scheme $2^{0}$ : Difference approximation of the second order of accuracy in space (6) and finite element scheme (12)-(14) in time;

Scheme 30: Finite element scheme with bicubic elements in space (7) and finite element scheme in time (12)-(14).

\section{STABILITY AND CONVERGENCE}

Let us examine the stability and accuracy of the schemes proposed. The scheme (10), (11) is known [2] to approximate problem (5) with second order of accuracy in step $\tau$; the scheme is known to be stable provided that the following conditions are satisfied

$$
D=D^{*}, \quad A=A^{*}>0, \quad D-1 / 4 \tau^{2} A \geq \varepsilon D, \quad 0<\varepsilon<1 .
$$

We write condition (13) as follows: $(1-\varepsilon) D-1 / 4 \tau^{2} A \geq 0,0<\varepsilon<1$. In view of bound (9), the last condition is satisfied for steps

$$
\tau \leq \frac{2}{\omega_{0}(1-\varepsilon)}
$$

We have the following result.

Theorem 1. Under condition (17), the solution by scheme $1^{0}$ converges to a sufficiently smooth solution of problem (1)-(3) and the following bound

$$
\|y(t)-u(t)\|_{A}+\left\|y_{t}(t)-u_{t}(t)\right\|_{D} \leq M\left(\tau^{2}+h^{2}\right)
$$

holds. Here $\|v\|_{D}=\sqrt{\left(D_{V}, v\right)}=\|v\|_{W_{2}^{1}\left(\omega_{h}\right)},\|v\|_{A}=\sqrt{(A V, v)}=\omega_{0}\left\|v_{x_{1}}\right\|_{L_{2}\left(\omega_{h}\right)}$ are norms in the space of grid functions $H_{h} ; y_{t}=\left(y^{n+1}-y^{n}\right) / \tau$.

Let us now formulate a result pertaining to the stability and accuracy of scheme (12)-(14).

Theorem 2 [4-6]. If $A^{*}=A>0, D^{*}=D>0$ and

$$
\alpha-\beta=1 / 12, \quad D-\delta \tau^{2} A \geq \varepsilon D, \quad 0<\varepsilon<1, \quad \delta=\max \{\alpha, \beta, 1 / 12,0\},
$$

then the solution $y(t)$ by scheme (11), (12) converges to the solution of problem (5) with $u_{h}(t) \in C^{6}[0, T]$, and the following bounds hold:

$$
\left\|u_{h}(t)-y(t)\right\|_{A}+\left\|\dot{u}_{h}(t)-\dot{y}(t)\right\|_{D} \leq M \tau^{4} .
$$

The proof is based on a reduction of the two-layer vector scheme (12)-(14) to the three-layer one separately for the solution $y$ and for its derivative $\dot{y}$. Condition (18) for values of parameters $\alpha=1 / 8, \beta=$ $1 / 24$, and hence $\delta=1 / 8$, leads us to the following limitation regarding the time step: 


$$
\tau \leq \frac{2 \sqrt{2}}{\omega_{0}(1-\varepsilon)}
$$

Hence, the stability condition enables us to use $\sqrt{2}$ times as many steps for the finite element method (12)-(14) as compared to that of the difference scheme (10), (11).

To evaluate the accuracy of schemes $2^{0}$ and $3^{0}$, it is also necessary to evaluate the error $z=u_{h}-u$. The final results will be formulated in accordance with the approach of the theory of difference schemes [2] and of the theory of the finite element method [3].

Theorem 3. Assume that the stability condition (19) for scheme $2^{0}$ is satisfied. Then, the solution $y(t)$ converges to a sufficiently smooth solution of problem (1)-(3) and the following bounds hold:

$$
\|u(t)-y(t)\|_{1} \leq M\left(h^{2}+\tau^{4}\right) .
$$

Theorem 4. Assume that the stability condition (19) for scheme $3^{0}$ is satisfied. Then, the solution $y(t)$ converges to a sufficiently smooth solution of problem (1)-(3) and the following bound holds:

$$
\|u(t)-y(t)\|_{1}+\|\dot{u}(t)-\dot{y}(t)\|_{1} \leq M\left(h^{3}+\tau^{4}\right) .
$$

\section{ALGORITHMS FOR THE SCHEMES}

Schemes (10) and (11) were implemented as follows:

$$
\begin{gathered}
y^{n+1}=2 y^{n}-y^{n-1}+\tau^{2} D^{-1}\left(f_{h}\left(t_{n}\right)-A y^{n}\right), \quad n=1,2, \ldots, \\
y^{1}=y^{0}+\tau u_{1, h}+\frac{\tau^{2}}{2} D^{-1}\left(f_{h}^{0}-A y^{0}\right) .
\end{gathered}
$$

The inverse matrix, corresponding to operator $D$ (in what follows, we shall not distinguish between an operator and the corresponding matrix), was calculated once on the first layer through the Cholesky method (the square root algorithm). On the subsequent layers, the solution was calculated by multiplying $D^{-1}$ by the vector on the right-hand side. The algorithm for scheme (12)-(14) amounts to solving two equations (see $[4,5])$

$$
C y^{n+1}=F_{1}, \quad C \dot{y}^{n+1}=F_{2}
$$

on each of the layers; here, $C=D^{2}-(\alpha-1 / 6) \tau^{2} A D+(\alpha / 12-\beta / 4) \tau^{4} A^{2}$, and the right-hand sides are calculated from the available $y^{n}, \dot{y}^{n}$ and $\Phi_{1}, \Phi_{2}$.

Matrix $C$ is factorized into a product $C=C_{1} C_{2}=\left(D-\omega_{1} A\right)\left(D-\omega_{2} A\right)$, where $\omega_{1}$ and $\omega_{2}$ are the roots of the equation

$$
\omega^{2}+(\alpha+\gamma-1 / 4) \omega+\alpha \gamma-\beta / 4=0 .
$$

For $\alpha=1 / 8, \beta=1 / 24$, we have, for example, $\omega_{1}=-1 / 24, \omega_{2}=0$.

It follows that $y^{n+1}=C^{-1} F_{1}=C_{2}^{-1} C_{1}^{-1} F_{1}, \dot{y}^{n+1}=C_{2}^{-1} C_{1}^{-1} F_{2}$. To invert matrices $C_{1}$ and $C_{2}$, the direct method of square roots was applied once at the initial time instant. On the remaining layers, the solution was found by multiplying the matrix $C^{-1}=C_{2}^{-1} C_{1}^{-1}$ by vectors $F_{1}$ and $F_{2}$.

Most of the operations for scheme (10), (11) are taken to invert the single operator $D$ on the first layer and for the subsequent multiplication of vector $f_{h}^{n}-A y^{n}$ by $D^{-1}$ on the remaining layers.

For scheme (11), we invert two operators $C_{1}=D-\omega_{1} A$ and $C_{2}=D-\omega_{2} A$ on the first layer. On the remaining layers, we multiply vectors $F_{1}$ and $F_{2}$ by $C^{-1}=C_{2}^{-1} C_{1}^{-1}$. Consequently, implementation of scheme (12) - (14) takes approximately four times as many operations as compared with scheme (10), (11).

The increase in the computational burden for scheme $3^{0}$ is also due to constructing the stiffness matrices $\mathbf{D}, \mathbf{A}$ (see (8)), which is done using numerical integration (once, at the initial instant $t=0$ ). For schemes $1^{0}$ and $2^{0}$, the matrices of operators $D$ and $A$ are constructed explicitly. 
Table

\begin{tabular}{c|l|r|r|r}
\hline$k$ & $N_{1} / N_{2}$ & Scheme $1^{0}$ & \multicolumn{1}{|c|}{ Scheme $2^{0}$} & Scheme $3^{0}$ \\
\hline 1 & $10 / 5$ & -0.009365 & -0.008792 & 0.000684 \\
2 & $20 / 10$ & -0.002724 & 0.002102 & -0.000296 \\
3 & $40 / 20$ & -0.001143 & -0.000527 & -0.000065 \\
\hline
\end{tabular}

\section{TEST}

Consider the following problem: Solve Eq. (1) with inhomogeneous boundary-value conditions of the first kind on one of the sides of the rectangle $\bar{\Omega}: u\left(0, x_{2}, t\right)=x_{2}\left(l_{2}-x_{2}\right) \equiv \mu\left(x_{2}\right) ; u=0, x \in \Gamma /\left(x_{1}=0\right)$; the initial conditions are as follows: $u=\frac{\partial u}{\partial t}=0$ at $t=0$. In order to reduce this problem to the initial problem (1)-(3), we define the function $\varphi_{0}\left(x_{1}, x_{2}\right)=\frac{l_{1}-x_{1}}{l_{1}} \mu\left(x_{2}\right)$. Then, for function $v=u-\varphi_{0}\left(x_{1}, x_{2}\right)$, we obtain a problem with a homogeneous equation of form (1) with homogeneous boundary data and initial conditions

$$
t=0: \quad v=-\varphi_{0}\left(x_{1}, x_{2}\right), \quad \frac{\partial v}{\partial t}=0
$$

Solving this problem by the method of the separation of variables, we obtain

$$
\begin{aligned}
u\left(x_{1}, x_{2}, t\right)=\varphi_{0}\left(x_{1}, x_{2}\right)-\frac{16 l_{2}^{2}}{\pi^{4}} \sum_{k=1}^{\infty} \sum_{p=1}^{\infty} \frac{1}{k(2 p-1)^{3}} \cos \left(\omega_{0} \sqrt{\zeta_{\text {crit }}} t\right) \\
\times \sin \left(\frac{k \pi x_{1}}{l_{1}}\right) \sin \left(\frac{(2 p-1) \pi x_{2}}{l_{2}}\right),
\end{aligned}
$$

where $\zeta_{\text {crit }}=1 /\left(1+(k /(2 p-1))^{2}\left(l_{1} / l_{2}\right)^{2}\right)$.

The exact solution at point $x_{1}=1.0$ and $x_{2}=0.5$ at time $t=3$ was calculated using formula (20), and is as follows: $u=0.158814$. The infinite series was replaced by a finite sum with $k=\overline{1,5000}$ and $p=\overline{1,40}$. In doing so, the error in calculating the solution at the initial instant was $10^{-6}$ (the large number of terms of the series required for this accuracy is due to the fact that the solution in not smooth at the initial instant of time $t=0$ ).

We chose the following parameters of the problem $\omega_{0}=2, l_{1}=2$, and $l_{2}=1$; a step in time is $\tau=0.1$ and the number of divisions in the space variables varies. The parameters of scheme (12)-(14) were as follows: $\alpha=1 / 8, \beta=1 / 24$.

Table 1 shows errors $z=y-u$ of the numerical solution for various schemes for various space grids at point $x_{1}=1.0, x_{2}=0.5$ at time $t=5$. The number of the scheme is shown in the column number and the row number corresponds to different grids.

This table shows the advantage of scheme $3^{0}$ over schemes $1^{0}$ and $2^{0}$ in terms of accuracy. The advantage of scheme $2^{0}$ over scheme $1^{0}$ is realized for sufficiently small space grid meshes, $N_{1}=40$ and $N_{2}=20$; this is because the error in rough space meshes makes up the principal part of the error of the method. As regards the computational burden (we have already pointed out that it is approximately higher by a factor of 4 for scheme $3^{0}$ than for scheme $1^{0}$ ), it is worth noting that even with increasing the total number of nodes by a factor of 16 (see Table $1, k=3, N_{1} / N_{2}=40 / 20$ ) scheme $1^{0}$ fails to reach the accuracy of scheme $3^{0}$ (see Table $1, k=1, N_{1} / N_{2}=10 / 5$ ).

It is worth noting that solving problems with arbitrary inhomogeneous boundary data of the first kind by means of scheme $3^{0}$ involves difficulties (shared by all finite element problems involving space variables) in choosing a function $\varphi_{0}\left(x_{1}, x_{2}\right)$ to satisfy these conditions. In such a case one may consider using scheme $2^{0}$, since these conditions can be easily approximated by means of the grid method.

Scheme $3^{0}$ was also used to solve problem (1), (2) with the right-hand side $f=$ $\sin \left(\pi x_{1} / l_{1}\right) \sin \left(\pi x_{2} / l_{2}\right) \sin (\omega t)$ and to furnish a solution of the problem in questions. For this problem, the 


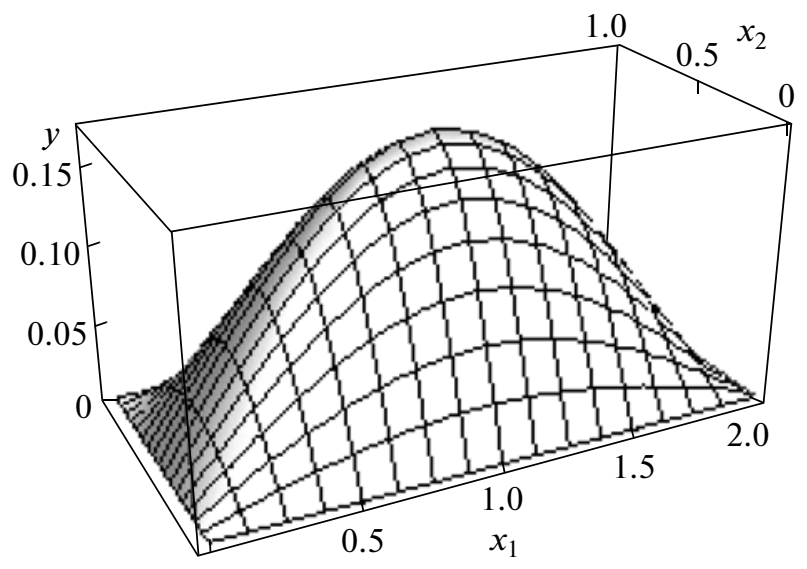

Fig. 1.

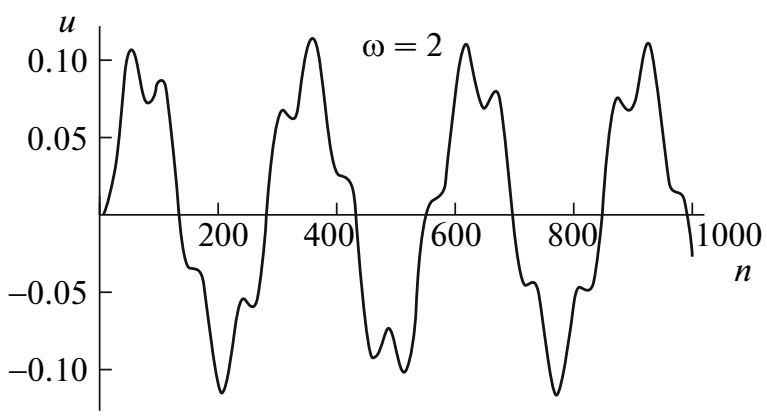

Fig. 3.

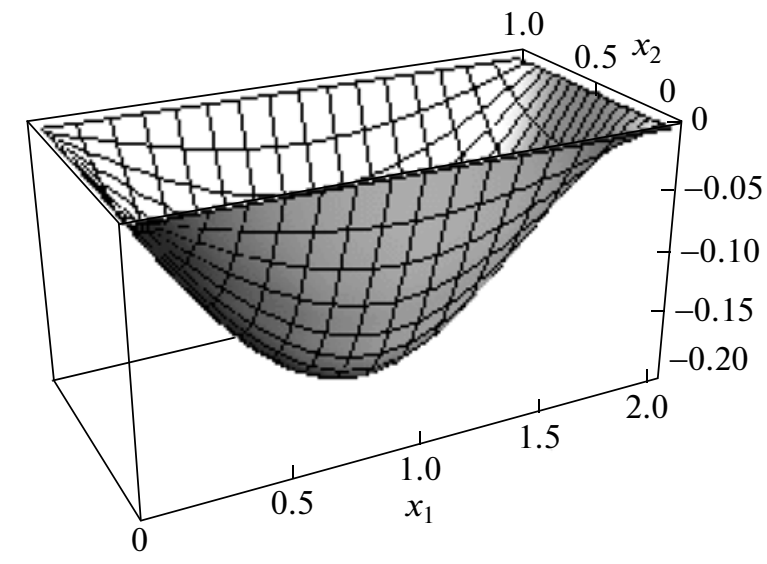

Fig. 2.

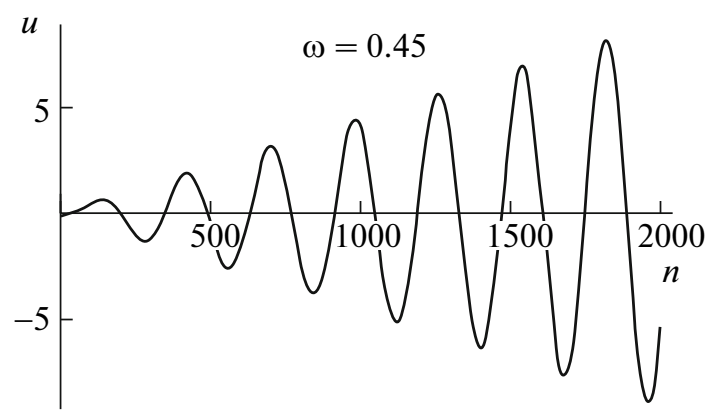

Fig. 4.

following parameters were chosen: $\omega_{0}=1, l_{1}=2$, and $l_{2}=1$, and the frequencies were as follows: $\omega=0.2$ and $\omega=2$. For the Cauchy problem, the steady solution is either of the hyperbolic type (for $\omega<\omega_{0}$ ) or the elliptic type (for $\omega>\omega_{0}$ ) [1, p. 124]. For the boundary-value problem in question, the calculations show that the steady-state solutions were similar in their behavior: the form of the solutions replicates the form of the right-hand side in the space variables, and the sign of the solution changes at different instants of time (see Figs. 1 and 2). The change in sign of the solution is clear from Fig. 3, which illustrates the graph of the behavior in time ( $n$ is the step number in time) of the position of the middle point of the domain, $x_{1}=l_{1} / 2=1$ and $x_{2}=l_{2} / 2=0.5$ for $\omega=2$.

This substantiates the conclusion of [1] that there is no difference in the behavior of the solutions for different $\omega$ for boundary-value problems for a weakly stratified fluid.

A value $\omega=0.45$ is found at which resonance of the external force with one of the eigenfrequencies of the fluid inside a closed volume takes place, see Fig. 4.

\section{CONCLUSIONS}

In this paper we worked out and examined a highly accurate method for solving the problem of internal wave fluid dynamics. This method is based on the finite element approximation in space and time by means of polynomials of the third degree. To implement this method we developed an algorithm for which a test on the exact solution given in the form of a Fourier series was conducted and a comparison with the finite difference method was performed. The method proposed has been used to solve the problem of steady-state oscillations of a weakly stratified fluid. 


\section{REFERENCES}

1. S. A. Gabov and A. G. Sveshnikov, Linear Problems in the Theory of Non-Steady-State Internal Waves (Nauka, Moscow, 1990) [in Russian].

2. A. A. Samarskii, Theory of Difference Schemes (Nauka, Moscow, 1977) [in Russian]

3. G. I. Marchuk and V. I. Agoshkov, Introduction to Projection Lattice Methods (Nauka, Moscow, 1981) [in Russian].

4. M. N. Moskalkov and D. Utebaev, "Investigation of Difference Schemes of Finite Element Method for SecondOrder Unsteady-State Equations,” J. Comput. Appl. Math., No. 92, 70-76 (2005).

5. M. N. Moskal'kov, "Scheme of the High-Accuracy Finite Element Method for Solving Non-Steady-State Second-Order Equations," Differ. Uravn. 16 (7), 1283-1292 (1980).

6. D. Utebaev, "Numerical Solution to the Linear Unsteady-State Problems in the Theory of Internal Waves," Dokl. Akad. Nauk Resp. Uzbekistan, Ser. Mat. Tekh. Nauki, Estestvoznanie, No. 3, 49-53 (2008). 